\title{
Injury to the superior vena cava during hemodialysis: pitfalls associated with multi-lumen catheters
}

\author{
Shinichi Ijuin ${ }^{1}$, Satoshi Ishihara ${ }^{1}$, Masafumi Fukushima ${ }^{1}$, Daigo Fujiwara ${ }^{1}$, Masafumi \\ Suga $^{1}$, Shota Kikuta ${ }^{1}$, Akihiko Inoue ${ }^{1}$, Shigenari Matsuyama ${ }^{1}$, Tetsunori Kawase ${ }^{1}$, and \\ Shinichi Nakayama ${ }^{1}$ \\ ${ }^{1}$ Hyogo Emergency Medical Center
}

May 21, 2020

\begin{abstract}
We report a case of perforation of the superior vena cava caused by a multi-lumen central venous catheter. Diagnosis was delayed because extra-corporeal blood flow was not interrupted. Clinicians should be aware of potential vascular complications associated with multi-lumen catheters even in cases in which hemodialysis continues with no complications.
\end{abstract}

\section{Key words:}

blood purification, innominate vein, triple lumen catheter

\section{Key Clinical Message:}

The clinicians should be careful while a triple-lumen catheter is used as a vascular access for blood purification, even if a catheter is penetrated the vascular wall, the bloodstream may be maintained.

\section{Introduction}

A multi-lumen catheter may be used for vascular access during the continuous renal replacement therapy (CRRT). While this is a commonplace procedure, it may be associated with serious and even fatal complications. We report here a case of vascular injury associated with the insertion of a triple lumen catheter; diagnosis was delayed because CRRT could be continued despite this serious injury.

\section{Case presentation}

An 87-year-old woman was transported to our institute for evaluation and treatment of an acute disturbance of consciousness and right hemiplegia. Her medical history was notable for chronic renal failure due to diabetic nephropathy that required regular hemodialysis (HD). Upon admission, she was diagnosed with cerebral infarction by computed tomography $(\mathrm{CT})$ and magnetic resonance image (MRI) that was treated via a thrombectomy. Given her clinical condition, we were concerned that conventional HD would be associated with a high risk of disequilibrium syndrome; as such, CRRT was recommended. An initial effort was made to obtain central access via insertion of a triple-lumen catheter into the right internal jugular vein; this effort failed due to accidental arterial puncture.

On day 2, we inserted a triple-lumen catheter (Gentle Cath@ COVIDIEN) via the left internal jugular vein under fluoroscopic guidance (Fig. 1). The catheter advanced easily and was secured with sutures. Appropriate positioning of the tip was confirmed via blood withdrawal/regurgitation and saline flushes through all three of the catheter lumens. After initiation of CRRT, a chest radiograph taken on day 3 revealed an enlarged mediastinum; at that time, vital signs remained within normal limits. CT was performed on day 
6; this examination revealed mediastinitis which suggested the possibility of a vascular injury associated with catheter insertion (Fig. 2). However, we were able to continue with CRRT without issues or difficulty. Transcatheter angiography performed on day 7 revealed extravasation of the contrast media from the proximal lumen, a finding that was consistent with vascular injury (Fig. 3); as such, an emergency thoracotomy was performed. During the procedure, we determined that the infusion port of the catheter had penetrated one wall of superior vena cava (SVC); both drainage and return ports remained inside vessel (Fig. 4). The catheter was removed and the site of perforation was sutured. Her postoperative course was uneventful, and she was transferred to a rehabilitation hospital on 20 day after surgery.

\section{Discussion}

Central venous catheters (CVCs) are frequently associated with complications, notably

when placed on the patient's left side. ${ }^{1-3}$ Abdelkefi et al. ${ }^{3}$ reported that the catheter tip is often positioned so that it is in direct contact with the lateral wall of the SVC where the innominate vein is at a right angle to the SVC. Several mechanisms of vascular injury due to insertion of CVCs have been reported, including (1) direct trauma during insertion of the guide-wire or catheter, (2) the movement of the catheter tip after insertion due to changes in arm, neck and/or head position, (3) continuous contact of the catheter tip with vascular wall associated with the heartbeat, and (4) the vascular endothelial damage due to infusion of a hyperosmolar solution through the distal port. ${ }^{4-6}$

In this case, we speculate that the catheter perforated the vessel wall due to continuous contact of the tip with the vessel and movement associated with a change in neck and head position. The perforation was unlikely to have occurred during insertion; positioning of the catheter was confirmed at that time by evaluation of blood regurgitation and saline flushes through all three lumens. Moreover, only crystalloid fluids were introduced via distal port; as such, the perforation was not likely to be related to infusion of hyperosmolar solutions.

A chest radiograph performed after initiation of CRRT revealed an enlarged mediastinum; this finding suggested the possibility of vascular injury, although we misdiagnosed it as mediastinitis associated with the first unsuccessful attempt at catheter insertion. At that time, CRRT continued with no difficulties: this observation led to the initial misdiagnosis in this case. Ultimately, we determined that both the drainage and return ports were remained within the vessel. To our knowledge, this is the first documented case of the vascular injury associated with multi-lumen catheters in which hemodialysis could be continue. Clearly, had we checked to determine appropriate blood regurgitation from the infusion port, this condition would have been diagnosed earlier.

\section{Conclusion}

When using a multi-lumen catheter for central access during CRRT, clinicians should be careful not to miss the possibility of vascular injury even in cases which blood flow through an extracorporeal circuit could be maintained. A thorough understanding of the structure of the CVC might prevent similar delayed diagnoses.

\section{Conflict of interest}

The authors declare no conflict of interest.

\section{Author Contribution:}

S. Ijuin: involved in main work, data collection, and manuscript writing.

MF, DF, MS, SK, AI, SM, TK, and SN: involved in data collection, manuscript revision.

S. Ishihara: involved in final revision

\section{Acknowledgement}

None

\section{References}


1. Sainarcsh VV, Jain SH, Patel HV, et al:Malplacement of hemodialysis permcatheter into anterior mediastinum following innominate vein perforation. Semin Dial.2012;25(1):95-6.

2. Mukau L, Talamini MA, Sitzmann JV, et al:Risk factors for central venous catheter-related vascular erosions. JPEN J Parenter Enteral Nutr. 1991;15(5):513-516

3. Abdelkefi A, Ben Gaied O, Ladeb S, et al:Perforation of the superior vena cava after subclavian catheterization; a rare complication after autologous PBSCT. Bone Marrow Transplant. 2009;43(11):891-892.

4. Booth SA, Norton B, Mulvey DA:Central venous catheterization and fatal cardiac tamponade. Br J Anaesth. 2001;87:298-302

5. Vesely TM:Central venous catheter tip position: a continuing controversy. J Vasc Interv Radiol. 2003.14:527-34.

6. Vesely TM, Beathard G, Ash S, et al.:Classification of complications associated with hemodialysis vascular access procedures. A position statement from the American Society of Diagnostic and Interventional Nephrology. J Vasc Access. 2008; 9: 12-9.

\section{Figure legends}

Figure 1

Perspective image after insertion of a triple-lumen catheter.

Arrow indicates the catheter tip positioned at the superior vena cava.

Figure 2

Enhanced CT scan revealed a fluid collection and air in the mediastinum.

Arrows indicates catheter placed at the superior vena cava.

Figure 3

Transcatheter angiography through the triple-lumen catheter revealed extravasation of the contrast agent.

Arrow indicates extravasation of contrast agent detected outside of the SVC within the mediastinum.

Figure 4

Schema presenting of our hypothesis regarding the mechanisms underlying SVC perforation in this patient.

The catheter perforated the wall of the SVC. The drainage and return ports remained within the vessel; only the infusion port was detected outside the SVC.

CCV: Common carotid vein

SVC: Superior vena cava

PV: Pulmonary vein 
Figure 1

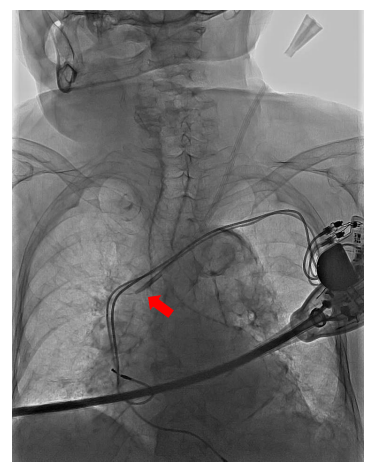

\section{Hosted file}

BA catheter rup.pdf available at https://authorea.com/users/324742/articles/452867-injuryto-the-superior-vena-cava-during-hemodialysis-pitfalls-associated-with-multi-lumencatheters 